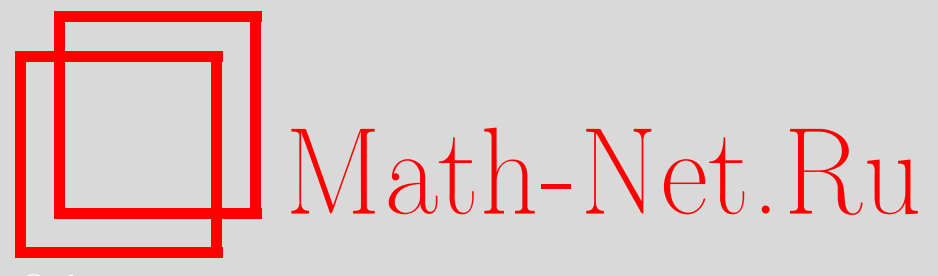

А. А. Грушо, О существовании скрытых каналов, Дискрет. матем., 1999, том 11, выпуск 1, 24-28

DOI: https://doi.org/10.4213/dm363

Использование Общероссийского математического портала Math-Net.Ru подразумевает, что вы прочитали и согласны с пользовательским соглашением http://www.mathnet.ru/rus/agreement

Параметры загрузки:

IP : 35.173 .219 .12

26 апреля 2023 г., 14:00:59 


\title{
О существовании скрытых каналов
}

\author{
(C) 1999 г. $\quad$ A. A. Грушо
}

\begin{abstract}
Устанавливаются условия, при которых существует канал связи между двумя субъектами, скрытый от контролирующего субъекта.

Работа выполнена при поддержке Российского фонда фундаментальных исследований, проект 97-01-00162.
\end{abstract}

В работе рассматривается следующая задача. Имеются две пары пользователей или субъектов компьютерной системы, $\left(U_{1}, U_{2}\right)$ и $\left(U_{1}^{\prime}, U_{2}^{\prime}\right)$, которые используют один канал связи (без помех) для передачи информации. Для простоты полагаем, что передача информации допускается только в одном направлении, от $U_{1}$ к $U_{2}$ или от $U_{1}^{\prime}$ к $U_{2}^{\prime}$, в этих случаях мы пишем $U_{1} \mapsto U_{2}$ или $U_{1}^{\prime} \mapsto U_{2}^{\prime}$. Вторая пара пытается организовать передачу своей информации по каналу $U_{1}^{\prime} \mapsto U_{2}^{\prime}$ так, чтобы внешне она не отличалась от передачи по каналу $U_{1} \mapsto U_{2}$ (построить скрытый канал [1]). Для того чтобы формализовать задачу, введем некоторый контролирующий субъект $U$, который наблюдает за передачей, но не знает, какая пара субъектов, $\left(U_{1}, U_{2}\right)$, или $\left(U_{1}^{\prime}, U_{2}^{\prime}\right)$, ее ведет. Предполагается, что объем передаваемой информации велик, так что $U$ реально может лишыь наблюдать за выходом некоторого автомата $A$, который перехватывает всю передачу, обрабатывает ее и результат выдает субъекту $U$ в виде некоторого сообщения $y$. Предполагается, что субъекты $\left(U_{1}, U_{2}\right)$ и $\left(U_{1}^{\prime}, U_{2}^{\prime}\right)$ знают схему контроля и автомат $A$ и могут использовать эти данные для организации скрытого от $U$ канала $U_{1}^{\prime} \mapsto U_{2}^{\prime}$. Возможность существования скрытого канала $U_{1}^{\prime} \mapsto U_{2}^{\prime}$ будем исследовать в рамках вероятностной модели так же, как раньше исследовался вопрос о возможности скрытого управления Троянским конем в компьютерной системе [2].

Пусть $X$ - множество сообщений, которые могут передаваться от $U_{1}$ к $U_{2}$ и $\mathbf{P}_{X}(x)$ - распределение вероятностей на $X$, при помощи которого мы моделируем канал $U_{1} \mapsto U_{2}$. Автомат $A$, обрабатывающий перехватываемые сообщения для контролирующего субъекта $U$, описывается набором $(X, S, Y, d, l)$, где $X$ - множество входных сообщений, совпадающцее с множеством возможных сообщений канала $U_{1} \mapsto U_{2}, S=\{1,2, \ldots, m\}$ - множество внутренних состояний автомата $A$, $Y=\left\{y_{1}, \ldots, y_{n}\right\}-$ множество сообщений, доступных субъекту $U, d: X \times S \rightarrow S-$ функция переходов, функция $l: X \times S \rightarrow Y$ определяет выходное сообщение $y$ из $Y$ на основе обработки автоматом $A$ входного сообщения $x$, если в момент поступления сообщения $x$ автомат находился в состоянии $s \in S$. Таким образом, наблюдая канал $U_{1} \mapsto U_{2}$ в состоянии $s$ автомата $A$, субъект $U$ может получить сообщение $y$ из множества $Y$, выбранное в соответствии с вероятностной мерой

$$
Q_{s}(y) \doteq \mathbf{P}_{X}\{x: l(x, s)=y\} .
$$


Для описания канала $U_{1}^{\prime} \mapsto U_{2}^{\prime}$ рассмотрим множество $Z$ возможных сообщений от $U_{1}^{\prime}$ к $U_{2}^{\prime}$. Выбор сообщения $z$ из $Z$ определяется некоторой вероятностной мерой $\mathbf{P}_{Z}(z)$. Для сообщения $z$ выбирается некоторое сообщение $x$ из $X$ и передается по каналу. На приемном конце по $x$ восстанавливается $z$. Полагается, что на приемном конце в соответствии с некоторой неизвестной для $U$ договоренностью однозначно определяется, работает канал $U_{1} \mapsto U_{2}$ или $U_{1}^{\prime} \mapsto U_{2}^{\prime}$.

Рассмотрим следующий способ выбора сообщения $x$ по $z$. Принимающая и передающая стороны имеют функцию

$$
g: Z \times \Theta \rightarrow X
$$

где $\Theta$ - некоторое конечное множество. Однозначность восстановления сообщения $z$ определяется условием, что для любых $z \in Z$ и $\theta \in \Theta$

$$
g^{-1}(g(z, \theta))=z
$$

Выбор $\theta$ при заданном $z$ определяется условным распределением $\mathbf{P}_{\Theta}(\theta \mid z)$. Тогда распределение $\mathbf{P}(z, \theta)$ на $Z \times \Theta$ и функция $g(z, \theta)$ определяют вероятностную меру $\mathbf{P}_{X}^{\prime}(x)$ на $X$. Меры $\mathbf{P}_{X}$ и $\mathbf{P}_{X}^{\prime}$, вообще говоря, различны. Функции $l(x, s), s=1, \ldots, m$, индуцируют на множестве $Y$ меры $Q_{s}^{\prime}(y)$. Только в случае различия $Q$ и $Q^{\prime}$ наблюдатель $U$ может различить каналы $U_{1} \mapsto U_{2}$ и $U_{1}^{\prime} \mapsto U_{2}^{\prime}$.

Определение 1. Субъект $U$ не видит в состоянии $s \in S$ автомата $A$ канал $U_{1}^{\prime} \mapsto U_{2}^{\prime}$ тогда и только тогда, когда для любого распределения $\mathbf{P}_{Z}(z)$ и для любого $y \in Y$

$$
Q_{s}^{\prime}(y)=Q_{s}(y)
$$

В этом случае мы будем также говорить, что канал $U_{1}^{\prime} \mapsto U_{2}^{\prime}$ невидим для $U$ в состоянии $s \in S$ автомата $A$.

Определение 2. Субъект $U$ не видит канал $U_{1}^{\prime} \mapsto U_{2}^{\prime}$ в слабом смысле (канал невидим для $U$ в слабом смысле), если субъект $U$ не видит канал $U_{1}^{\prime} \mapsto U_{2}^{\prime}$ в каждом состоянии $s \in S$ автомата $A$.

Определим отображение $l: X \rightarrow Y^{m}$, полагая

$$
l(x)=(l(x, 1), \ldots, l(x, m)), \quad x \in X
$$

Распределения $\mathbf{P}_{X}$ и $\mathbf{P}_{X}^{\prime}$ на $X$ с помощью функции $l(x)$ индуцируют меры $Q(\bar{y})$ и $Q^{\prime}(\bar{y})$ на $Y^{m}$.

Определение 3. Субъект $U$ не видит канал $U_{1}^{\prime} \mapsto U_{2}^{\prime}$ в сильном смысле (канал невидим для $U$ в сильном смысле), если при любом распределении $\mathbf{P}_{Z}(z)$

$$
Q(\bar{y})=Q^{\prime}(\bar{y})
$$

для любого $\bar{y} \in Y^{m}$.

Лемма 1. Если $U$ не видит ханал $U_{1}^{\prime} \mapsto U_{2}^{\prime}$ в силъном смъсле, то $U$ не видит этот каніал в слабом смысле. 
Доказательство. В самом деле, для всех $s \in S$ и всех $y \in Y$

$$
Q_{s}(y)=\sum Q(\bar{y})
$$

где суммирование ведется по всем $\bar{y} \in Y^{m}$, у которых $s$-я координата равна $y$. Тогда из равенства $Q(\bar{y})=Q^{\prime}(\bar{y})$ следует, что для любого $y \in Y$ и любого $s \in S$ справедливо равенство $Q_{s}(y)=Q_{s}^{\prime}(y)$. Лемма доказана.

Легко получить условия, при которых существует канал $U_{1}^{\prime} \mapsto U_{2}^{\prime}$, невидимый для $U$ в сильном смысле.

Теорема 1. Если для лтобого $\bar{y} \in Y^{m}$ такого, что $Q(\bar{y})>0$, выполняется условие

$$
\left|l^{-1}(\bar{y})\right| \geqslant|Z|,
$$

то существует канал $U_{1}^{\prime} \mapsto U_{2}^{\prime}$, невидимъй для $U$ в сильном смвсле.

Доказательство. В качестве $\Theta$ возьмем $Y^{m}$ и для каждого $\bar{y} \in Y^{m}$ такого, что $Q(\bar{y})>0$, определим произвольное инъективное вложение $x=g(z, \bar{y})$ множества $Z$ в $l^{-1}(\bar{y}) \subseteq X$. Это можно сделать, так как для любого $\bar{y} \in Y^{m}$ при условии, что $Q(\bar{y})>0$, справедливо неравенство $\left|l^{-1}(\bar{y})\right| \geqslant|Z|$.

В силу того, что при различных $y$ прообразы $l^{-1}(\bar{y})$ не пересекаются, выполняется требование (1), то есть по $x$ однозначно восстанавливается $\bar{y}$ такое, что $x$ принадлежит $l^{-1}(\bar{y})$. Поэтому в силу инъективности $g(z, \bar{y})=x$ при данном $\bar{y}$, по данному $x$ однозначно восстанавливается $z$.

Для любого $z \in Z$ при $Q(\bar{y})>0$ определим

$$
\mathbf{P}_{\Theta}(\bar{y} \mid z)=Q(\bar{y}) .
$$

Докажем, что $U$ не видит канал $U_{1}^{\prime} \mapsto U_{2}^{\prime}$ в сильном смысле. Для произвольного распределения $\mathbf{P}_{Z}(z)$ и для каждого $x \in X$

$$
\mathbf{P}_{X}^{\prime}(x)= \begin{cases}\mathbf{P}_{Z}(z) Q(\bar{y}), & \text { если } x \in l^{-1}(\bar{y}), \quad x=g(z, \bar{y}), \\ 0 & \text { в остальных случаях. }\end{cases}
$$

Тогда для любого $\bar{y} \in Y^{m}$

$$
Q^{\prime}(\bar{y})=\sum_{x \in l^{-1}(\bar{y})} \mathbf{P}_{X}^{\prime}(x)=Q(\bar{y})
$$

Теорема доказана.

Следствие 1. Пусть $A(y, s)=l^{-1}(y, s)$ для любых $y \in Y u$ любых $s \in S u$

$$
A\left(y_{i_{1}}, \ldots, y_{i_{m}}\right)=\bigcap_{s=1}^{m} A\left(y_{i_{s}}, s\right)
$$

тогда, если $Q\left(y_{i_{1}}, \ldots, y_{i_{m}}\right)>0 u$

$$
\left|A\left(y_{i_{1}}, \ldots, y_{i_{m}}\right)\right| \geqslant|Z|,
$$

то существует канал $U_{1}^{\prime} \mapsto U_{2}^{\prime}$, невидимый для $U$ в слабом смысле. 
Утверждение следствия вытекает из теоремы 1 и леммы 1.

Если $U$ не видит канал $U_{1}^{\prime} \mapsto U_{2}^{\prime}$ в сильном смысле, то этот канал устроен так, как описано в доказательстве теоремы 1.

Теорема 2. Пусть $\left(\Theta, g(z, \theta), \mathrm{P}_{\Theta}(\theta \mid z)\right)$ определяют организацию канала $U_{1}^{\prime} \mapsto U_{2}^{\prime}$, который $U$ не видит в силъном смысле и $g(z, \theta)$ удовлетворлет условию (1).

Tогдa

$$
\sum_{\theta} \mathbf{P}_{\Theta}(\theta \mid z)=Q(\bar{y})
$$

где сумма берется по всем $\theta$, удовлетворяющим условиям

$$
g(z, \theta)=x, \quad l(x)=\bar{y}
$$

это означает, что в качестве параметра $\theta$ мы можем выбрать $\bar{y} \in Y^{m}$.

Доказательство. При всех распределениях $\mathbf{P}_{Z}(z)$ справедливо равенство $Q^{\prime}(y)=$ $Q(y)$, поэтому

$$
\begin{aligned}
\mathbf{P}_{X}\left(l^{-1}(\bar{y})\right) & =\mathbf{P}_{X}^{\prime}\left(l^{-1}(\bar{y})\right) \\
& =\sum_{x \in l^{-1}(\bar{y})} \sum_{\theta \in \Theta} I(x=g(z, \theta)) \mathbf{P}_{Z}(z) \mathbf{P}_{\Theta}(\theta \mid z),
\end{aligned}
$$

где $I(A)$ - индикатор события $A$ и для всех $x$ в силу (1) может существовать не более одного $z$ такого, что $x=g(z, \theta)$. Отсюда следует, что при вырожденных распределениях, сосредоточенных на $z_{1} \neq z_{2}$ из $Z$

$$
\begin{aligned}
Q(\bar{y}) & =\sum_{x \in l^{-1}(\bar{y})} \sum_{\theta \in \Theta} I\left(x=g\left(z_{1}, \theta\right)\right) \mathbf{P}_{\Theta}\left(\theta \mid z_{1}\right), \\
& =\sum_{x \in l^{-1}(\bar{y})} \sum_{\theta \in \Theta} I\left(x=g\left(z_{2}, \theta\right)\right) \mathbf{P}_{\Theta}\left(\theta \mid z_{2}\right) .
\end{aligned}
$$

Из (1) и (4) получаем, что для всех $x \in l^{-1}(\bar{y})$

$$
\sum_{\theta \in \Theta} \mathbf{P}_{\Theta}(\theta \mid z)= \begin{cases}Q(\bar{y}), & \text { если существуют } z \in Z, \theta \in \Theta \text { такие, что } x=g(z, \theta), \\ 0 & \text { в противном случае. }\end{cases}
$$

Пусть $\theta_{1} \sim \theta_{2}$ тогда и только тогда, когда существуют $z_{1}, z_{2} \in Z$ такие, что

$$
x_{1}=g\left(z_{1}, \theta_{1}\right), \quad x_{2}=g\left(z_{2}, \theta_{2}\right) \text {, }
$$

и $x_{1}, x_{2} \in l^{-1}(\bar{y})$ при некотором $\bar{y} \in Y^{m}$. Тогда это отношение эквивалентности разбивает все множество $\Theta$ на классы, которые можно пронумеровать элементами $\bar{y} \in Y^{m}$ при $Q(\bar{y})>0$. Теорема доказана.

Таким образом, если $U$ не видит канал $U_{1}^{\prime} \mapsto U_{2}^{\prime}$ в сильном смысле, то можно считать, что этот канал построен способом, описанным в доказательстве теоремы 1. 


\section{Список литературы}

1. Schneier B. Applied Cryptography. Protocols, Algorithms, and Source Code in C. Wiley, New York, 1998.

2. Грушо А. А. Скрытые каналы и безопасность информации в компьютерных системах. Дискретная математика (1998) 10, №1, 3-9.

Статья поступила 11.01.1999. 